\title{
Post-COVID recovery: characteristics of chronically critically ill patients admitted to a long-term acute care hospital [version
}

\section{1; peer review: 1 approved, 1 approved with reservations]}

\author{
Meg Stearn Hassenpflug (DiD), Dale Jun1,2, David R. Nelson1, Tamas Dolinay (D)1,2 \\ ${ }^{1}$ Barlow Respiratory Hospital, Los Angeles, California, 90026, USA \\ 2UCLA David Geffen School of Medicine, Los Angeles, California, 90095, USA
}

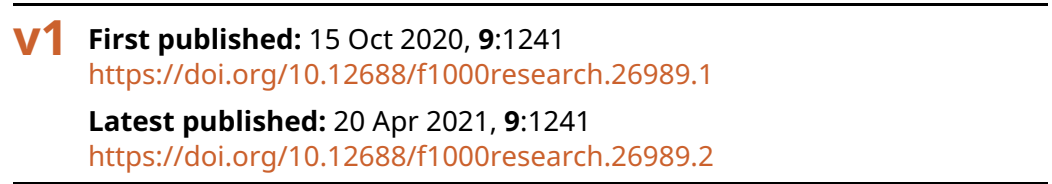

\section{Abstract}

Background: Survivors of COVID-19 pneumonia often suffer from chronic critical illness (CCI) and require long-term hospitalization. Long-term acute care (LTAC) hospitals are vital in the care of CCI patients, but their role for patients post COVID-19 infection is not known. Barlow Respiratory Hospital (BRH) is a 105-bed, LTAC hospital network serving ventilator-dependent and medically-complex patients transferred from the ICUs of hospitals in southern California. We report patient characteristics of our first series of COVID-19 survivors admitted to the post-acute venue of an LTAC hospital.

Methods: Single-center observational descriptive report of patients recovering from acute infectious complications of COVID-19 pneumonia requiring long-term respiratory support.

Results: From 28 April to 7 September 2020, 41 patients were admitted to BRH for continued recovery from COVID-19 pneumonia. The length of stay at the transferring hospital was twice that of nonCOVID patients admitted during the same time period. Median age: 68 [44-94] years, $61 \%$ male, $80.5 \%$ with tracheostomy, $51.2 \%$ on invasive mechanical ventilation, $22 \%$ receiving hemodialysis. All mechanical ventilation and hemodialysis interventions were initiated at the transferring hospital.

Conclusions: To our knowledge, this is the first report to characterize CCI and medically complex COVID-19 patients transferred to the postacute venue of an LTAC hospital. Patients on average spent over six weeks in the transferring hospital mostly in the ICU, are largely elderly, carry the known risk factors for COVID-19 infection, and experienced respiratory failure necessitating prolonged mechanical ventilation via tracheostomy. Our findings suggest that these patients will continue to require considerable medical interventions and treatments, including weaning from mechanical ventilation, owing to the numerous sequelae of the infection and the burden of acute-onchronic diseases. As ICU survival rates improve, this research further

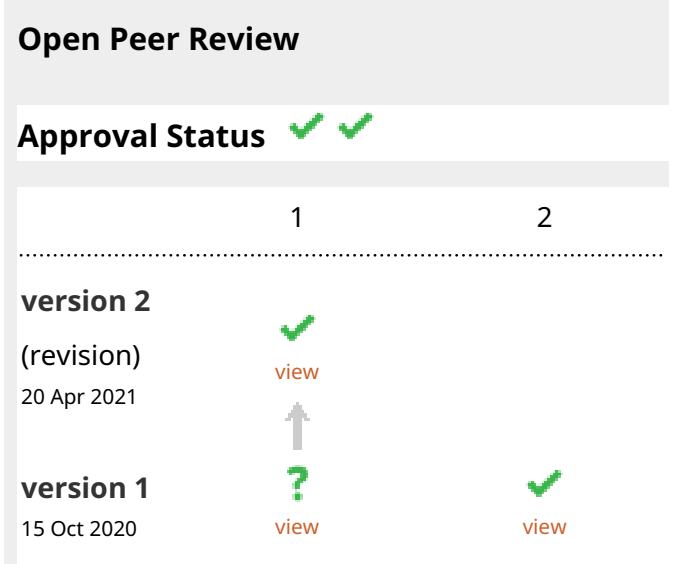

1. Anuj Mehta, Denver Health and Hospital Authority, Denver, USA

2. Anil Makam, University of California, San Francisco, San Francisco, USA

Any reports and responses or comments on the article can be found at the end of the article. 
emphasizes the important role of the LTAC hospital in responding to the COVID-19 crisis.

\section{Keywords}

COVID-19, post-acute, chronic critical illness, long-term acute care, mechanical ventilation, tracheostomy, recovery

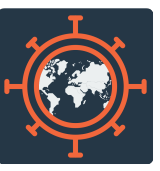

This article is included in the Emerging Diseases and Outbreaks gateway.

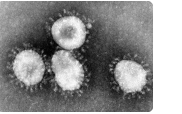

This article is included in the Coronavirus

collection.

\section{Corresponding author: Meg Stearn Hassenpflug (mhassenpflug@barlowhospital.org)}

Author roles: Hassenpflug MS: Data Curation, Formal Analysis, Investigation, Methodology, Project Administration, Writing - Original Draft Preparation, Writing - Review \& Editing; Jun D: Conceptualization, Investigation, Writing - Review \& Editing; Nelson DR: Resources, Validation, Writing - Review \& Editing; Dolinay T: Conceptualization, Formal Analysis, Investigation, Methodology, Project Administration, Supervision, Writing - Review \& Editing

Competing interests: No competing interests were disclosed.

Grant information: The author(s) declared that no grants were involved in supporting this work.

Copyright: ( $) 2020$ Hassenpflug MS et al. This is an open access article distributed under the terms of the Creative Commons Attribution License, which permits unrestricted use, distribution, and reproduction in any medium, provided the original work is properly cited.

How to cite this article: Hassenpflug MS, Jun D, Nelson DR and Dolinay T. Post-COVID recovery: characteristics of chronically critically ill patients admitted to a long-term acute care hospital [version 1; peer review: 1 approved, 1 approved with reservations] F1000Research 2020, 9:1241 https://doi.org/10.12688/f1000research.26989.1

First published: 15 Oct 2020, 9:1241 https://doi.org/10.12688/f1000research.26989.1 


\section{Introduction}

Advances in technology, research, and adoption of evidence-based practices have significantly improved intensive care unit (ICU) survivorship, creating the population of patients recognized as chronically critically ill $(\mathrm{CCI})^{1}$. This improved survival, however, is often accompanied by a prolonged and challenging course of recovery. This population now includes ICU survivors of coronavirus disease 2019 (COVID-19) in need of post-acute care for continued recovery from their infection. Long-term acute care (LTAC) hospitals are vital in the care of CCI patients ${ }^{2}$, but their role for patients post COVID-19 infection is not known. Barlow Respiratory Hospital (BRH) is a 105-bed, not for profit, LTAC hospital network serving ventilator-dependent and medically complex patients transferred from the ICUs of hospitals in southern California. Herein, we report patient characteristics of our first series of COVID-19 survivors admitted to the post-acute venue of an LTAC, as an essential step in the continuum of care for treatment, rehabilitation, and recovery.

\section{Methods}

\section{Study background}

This is a single-center observational descriptive report of patients recovering from acute infectious complications of COVID-19 pneumonia requiring long-term respiratory support. Over half were admitted on invasive mechanical ventilation having experienced respiratory failure at the transferring hospital. Patients were admitted for attempts at weaning from prolonged mechanical ventilation, as well as for continued care and treatment of infections, complications, and co-morbid conditions.

\section{Ethical considerations}

The study was approved by the Western Institutional Review Board (WIRB), reference: \#1-1348082-1. Only de-identified health information was collected and recorded in the database to ensure patient privacy and data safety. The WIRB waived the need for consent from patients who participated in the study.

\section{Data collection and statistical analysis}

Patients with at least one positive COVID-19 polymerase chain reaction testing (PCR) prior to admission to BRH were enrolled in the study on an ongoing basis. The tests were performed from nasopharyngeal, oropharyngeal or lower airway sampling. Exclusion criteria of the study was the absence of positive COVID-19 PCR testing prior to admission. This approach was followed to minimize biases in data collection. Due to the inherent false negative rate of the PCR testing, it is possible that we did not capture all previously COVID-19 positive patients ${ }^{3}$. Patients were determined to be in the post-infective phase prior to transfer to BRH.

The data are reported with binary values. The 0 represents absence and 1 represents presence of a condition (see Underlying data). Missing data are reported as unknown. Data were collected from our electronic medical record system using a combination of automated data extraction and manual collection. We collected baseline demographics (age, gender, race/ethnicity, premorbid location), presence of known COVID-19 risk factors, events at transferring hospital, and descriptors of status on admission to the LTAC to construct the Barlow COVID-19 data set.

All statistical analysis was performed using Microsoft Excel 2013 program (Microsoft Corporation, Santa Rosa, CA). We used descriptive statistics to describe the basic features of the data. Missing data were omitted from analysis. For the variables, serum albumin and serum glucose, $n=36$ and $n=40$ respectively. No statistical comparisons were made.

We used the STROBE cross sectional reporting guidelines to report this research ${ }^{4}$.

\section{Results}

Of 194 patients transferred to BRH from 28 April 2020 to 7 September 2020, 41 (21\%) were admitted for continued recovery from confirmed COVID-19 pneumonia. Selected demographics and patient characteristics are shown in Table 1.

Table 1. Selected demographics and characteristics of patients admitted for post-COVID recovery.

\begin{tabular}{|c|c|}
\hline Variable & $n=41$ \\
\hline Age, years (median [range]) & $\begin{array}{l}68 \\
{[44-94]}\end{array}$ \\
\hline Gender, male (\%) & 61 \\
\hline Premorbid location, home (\%) & 68.3 \\
\hline \multicolumn{2}{|l|}{ Race/ethnicity (n (\%)) } \\
\hline African American & $2(4.9)$ \\
\hline Asian/Pacific Islander & $6(14.6)$ \\
\hline Caucasian & $15(36.6)$ \\
\hline Hispanic & $18(43.9)$ \\
\hline \multicolumn{2}{|l|}{ COVID-19 risk factors (n (\%)) } \\
\hline Type 2 diabetes mellitus & $26(63.4)$ \\
\hline Hypertension & $31(75.6)$ \\
\hline Coronary artery disease & $11(26.8)$ \\
\hline Hyperlipidemia & $15(36.6)$ \\
\hline Obesity (BMI $\geq 30)$ & $16(39)$ \\
\hline \multicolumn{2}{|l|}{ At transferring hospital (n (\%)) } \\
\hline ARDS & $16(39)$ \\
\hline Sepsis/septic shock & $20(48.8)$ \\
\hline Invasive mechanical ventilation & $36(87.8)$ \\
\hline Tracheotomy & $33(80.5)$ \\
\hline Acute kidney injury/acute renal insufficiency & $21(51.2)$ \\
\hline Heart failure & $12(29.3)$ \\
\hline Transferring hospital ICU/CCU days (median [range]) & $38[8-77]$ \\
\hline $\begin{array}{l}\text { Transferring hospital length of stay, days (median } \\
\text { [range]) }\end{array}$ & $42[8-78]$ \\
\hline
\end{tabular}


The length of stay at the transferring hospital was twice that of non-COVID patients admitted during the same time period. All mechanical ventilation and hemodialysis interventions were initiated at the transferring hospital. Table 2 presents treatment interventions already in effect on admission to $\mathrm{BRH}$, descriptive characteristics, and laboratory values.

\section{Discussion and conclusions}

LTAC hospitals provide specialized care for patients suffering from $\mathrm{CCI}^{5}$. With increased survival in the ICU, the number of patients transferred to these hospitals has also increased in the past decades ${ }^{2}$. Early reports of the COVID-19 pandemic indicate that $5-12 \%$ of patients with COVID-19 infection require ICU hospitalization ${ }^{6-8}$. These numbers suggest that the role of LTAC hospitals will expand during the COVID-19 pandemic,

\section{Table 2. Status of post-COVID recovery patients on admission $(n=41)$.}

\begin{tabular}{|l|l|}
\hline Variable & $\mathbf{n}(\%)$ \\
\hline $\begin{array}{l}\text { Invasive mechanical } \\
\text { ventilation }\end{array}$ & $21(51.2)$ \\
\hline Admitted for weaning & $14(67)$ \\
\hline Tracheostomy tube & $33(80.5)$ \\
\hline Hemodialysis & $9(22)$ \\
\hline Enteral feeding tube & $32(78)$ \\
\hline Central line & $20(48.8)$ \\
\hline Indwelling urinary catheter & $17(41.5)$ \\
\hline Pressure injury $\geq$ stage 2 & $32(78)$ \\
\hline Multiple pressure injuries & $19(46.3)$ \\
\hline Laboratory values (mean (SD)) & \\
\hline Serum albumin (g/dl) & $2.82(0.61)$ \\
\hline Hematocrit (\%) & $30.0(5.8)$ \\
\hline BUN (mg/dl) & $40.1(26.3)$ \\
\hline Creatinine (mg/dl) & $1.43(1.85)$ \\
\hline Glucose (mg/dl) & $146.6(51.9)$ \\
\hline
\end{tabular}

due in part to their ability to treat patients with illnesses and conditions that do not follow a linear trajectory of improvement.

To our knowledge, this is the first report to characterize CCI and medically complex COVID-19 patients transferred to the post-acute venue of an LTAC hospital. Patients on average spent over six weeks in the transferring hospital mostly in the ICU, are largely elderly, carry the known risk factors for COVID-19 infection, and experienced respiratory failure necessitating prolonged mechanical ventilation via tracheostomy. Patients present with physiological imbalances, numerous penetrating and indwelling catheters and disruptions of skin integrity breaching host defenses, and manifestations of allostatic load burden.

Overall, our findings suggest that these patients will continue to require considerable medical interventions and treatments, including weaning from mechanical ventilation, owing to the numerous sequelae of the infection and the burden of acute-on-chronic diseases. As ICU survival rates improve, this research further emphasizes the important role of the LTAC in responding to the COVID-19 crisis. LTAC hospitals will play an increasingly critical function to fill gaps in our preparedness and response to COVID-19 infection by resuming and relieving care initiated in the acute hospital setting.

Our analysis is limited by several factors: it is a single center descriptive report, with a small cohort of patients, and a still emerging evidence base for COVID and post-COVID infection. Patient characteristics from this single center study may not be applicable to other centers or the post-COVID pneumonia population in general due to geographic differences in patient demographics, referral patterns, and facility-specific treatment capabilities. Efforts to quantify disease burden and report the number and variety of interventions may be warranted to objectify the intensity of treatment at the LTAC hospital. In addition to reporting broad outcomes (wean rate, length of stay, discharge disposition) a goal is to identify subgroups of patients and craft specific clinical outcomes.

\section{Data availability}

Open Science Framework: Database, https://doi.org/10.17605/ OSF.IO/VHJZG ${ }^{9}$. Registered $8^{\text {th }}$ October 2020 (https://osf.io/ 2c8q9).

Data are available under the terms of the Creative Commons Zero "No rights reserved" data waiver (CCO 1.0 Public domain dedication).
1. Nelson JE, Cox CE, Hope AA, et al: Chronic critical illness. Am J Respir Crit Care Med 2010: 182(4): 446-454.

PubMed Abstract | Publisher Full Text | Free Full Text

2. Kahn JM, Benson NM, Appleby D, et al.: Long-term acute care hospital utilization after critical illness. JAMA. 2010; 303(22): 2253-2259. PubMed Abstract | Publisher Full Text | Free Full Text

3. Woloshin S, Patel N, Kesselheim AS: False Negative Tests for SARS-CoV-2
Infection - Challenges and Implications. N Engl J Med. 2020; 383(6): e38. PubMed Abstract | Publisher Full Text

4. von Elm E, Altman DG, Egger M, et al.: The Strengthening the Reporting of Observational Studies in Epidemiology (STROBE) Statement: guidelines for reporting observational studies J Clin Epidemiol. 2008; 61(4): 344-9. PubMed Abstract | Publisher Full Text

5. Kahn JM, Werner RM, David G, et al.: Effectiveness of long-term acute care 
hospitalization in elderly patients with chronic critical illness. Med Care. 2013; 51(1): 4-10.

PubMed Abstract | Publisher Full Text | Free Full Text

6. Grasselli G, Pesenti A, Cecconi M: Critical Care Utilization for the CovID-19 Outbreak in Lombardy, Italy: Early Experience and Forecast During an Emergency Response. JAMA. 2020; 323(16): 1545-1545.

PubMed Abstract | Publisher Full Text

7. Young BE, Ong SWX, Kalimuddin S, et al.: Epidemiologic Features and Clinical
Course of Patients Infected With SARS-CoV-2 in Singapore. JAMA. 2020; 323(15): 1488-1494.

PubMed Abstract | Publisher Full Text | Free Full Text

8. Guan WJ, Ni ZY, Hu Y, et al.: Clinical Characteristics of Coronavirus Disease 2019 in China. N Engl J Med. 2020; 382(18): 1708-1720.

PubMed Abstract | Publisher Full Text | Free Full Text

9. Dolinay T, Jun, D, Hassenpflug MS, et al.: Database. 2020 http://wwww.doi.org/10.17605/OSF.IO/VHJZG 


\section{Open Peer Review}

\section{Current Peer Review Status:}

\section{Version 1}

Reviewer Report 15 February 2021

https://doi.org/10.5256/f1000research.29812.r77537

(C) 2021 Makam A. This is an open access peer review report distributed under the terms of the Creative Commons Attribution License, which permits unrestricted use, distribution, and reproduction in any medium, provided the original work is properly cited.

\section{Anil Makam}

Division of Hospital Medicine, San Francisco General Hospital and Trauma Center, Department of Medicine, University of California, San Francisco, San Francisco, CA, USA

The authors conducted a straightforward small descriptive study of COVID-19 patients, mostly who had CCI, who were transferred to a single non-profit LTACH in Southern California. The report describes baseline sociodemographic details and clinical characteristics at the preceding transferring hospital and upon admission. This small descriptive cohort study provides novel data that a small but meaningful population of COVID-19 patients experience CCI from COVID-19, and implies (but does not show) that they likely will suffer from post-ICU syndrome even after their LTACH stay. I have a few comments:

1. As the pandemic evolved, hospitals and clinicians gained experience in caring for this population. Were patients transferred later during the study period different that patients transferred earlier on?

2. Please provide the median LOS for non-COVID patients to support the claim that the preceding hospital LOS for COVID-19 patients was twice that of non-COVID-19 patients. Also, the interpretation of this is unclear, as COVID-19 patients may not have been transferred due to unfamiliarity with the disease course and isolation requirements, and not necessarily because of differences in severity/complexity of illness

3. What data were missing - just albumin and glucose? The sentence is awkwardly written, please correct.

4. Why were only 14 of 21 patients on invasive MV admitted for weaning? Were the other 7 chronically ventilated?

Is the work clearly and accurately presented and does it cite the current literature? Yes

Is the study design appropriate and is the work technically sound? 
Yes

Are sufficient details of methods and analysis provided to allow replication by others? Yes

If applicable, is the statistical analysis and its interpretation appropriate?

Yes

Are all the source data underlying the results available to ensure full reproducibility?

No

Are the conclusions drawn adequately supported by the results?

Yes

Competing Interests: No competing interests were disclosed.

Reviewer Expertise: LTACHs, health services research, comparative effectiveness, geriatrics, postacute care, policy.

I confirm that I have read this submission and believe that I have an appropriate level of expertise to confirm that it is of an acceptable scientific standard.

Author Response 12 Apr 2021

Meg Hassenpflug, Barlow Respiratory Hospital, Los Angeles, USA

We kindly thank the Reviewer for his thoughtful comments and suggestions for our paper. Our responses follow:

1. The Reviewer notes that as the pandemic evolved, hospitals and clinicians gained experience in caring for this population. Were patients transferred later during the study period different that patients transferred earlier on? Referring to the study inclusion dates, our paper reflects admissions during roughly a four month time period. In view of the brief snapshot in time, along with a small cohort of patients we did not attempt to compare time periods at this point. We agree that such a comparison would be of interest and we will explore three, four, and six month time intervals as we build on this effort.

2. An oversight on our part in the Abstract and Results is that we failed to include the actual data on length of stay (LOS) at the transferring acute care hospital. We regret the omission and have excluded the related statement from the Abstract, and added the data to Results in the revised text. The LOS at the transferring acute care hospital was median 42 [8-78] days for the post-COVID pneumonia cohort, and median 16 [196] days for non-COVID patients admitted during the same time period. We made no attempt to interpret the data, and considered this simply an interesting informational contrast to be made at that point in time.

3. Regarding missing data: The Reviewer notes that the statements regarding missing 
data and the various " $n$ " for the laboratory values serum albumin and serum glucose were perhaps awkwardly written. We were informing the reader that these two variables presented in Table 2 were not reflective of the entire cohort of 41 patients due to a few missing values. The text has been revised.

4. The Reviewer observes that just 14 of the 21 patients on invasive mechanical ventilation were admitted for weaning. He poses the question on whether the other seven patients were chronically ventilated. We welcome the question and thank the Reviewer for his observation. None of the seven patients excluded from weaning were chronically ventilated prior to admission to the transferring hospital. Upon evaluation by the consulting pulmonologist on admission to our LTAC, patients were determined not to be weaning candidates for the following reasons: physiologic instability (unmet readiness to wean parameters), poor mentation or neurocognitive disorders. We have now included this information in the revised text. Thank you so much for the question.

Competing Interests: No competing interests were disclosed.

Reviewer Report 08 February 2021

https://doi.org/10.5256/f1000research.29812.r77597

(C) 2021 Mehta A. This is an open access peer review report distributed under the terms of the Creative Commons Attribution License, which permits unrestricted use, distribution, and reproduction in any medium, provided the original work is properly cited.

\section{Anuj Mehta}

Division of Pulmonary, Critical Care, and Sleep Medicine, Department of Medicine, Denver Health and Hospital Authority, Denver, CO, USA

Thank you for allowing me the opportunity to review "Post-COVID recovery: characteristics of chronically critically ill patients admitted to a long-term acute care hospital" for consideration for your journal. The authors present an early case series of patients in the post-COVID-19 state who were admitted to an LTAC. They describe a significant burden of disease that highlights the need for ongoing care after initial recovery from acute COVID-19 illness. The authors present the experience of Barlow Respiratory Hospital which is a unique and large LTAC with a large referral base. The primary strength of the manuscript is that it presents novel data and gives a picture of the ongoing burden of disease related to COVID-19. However, the two major gaps are a sense of outcomes from the LTACH (which may be difficult to quantify as some patients may still be receiving treatment) and a sense of context with comparisons to the prior year. I have listed my specific concerns below. The source data is not included but is presented in an appropriate manner.

\section{Major Comments}


1. The question that naturally stems from the authors description is what happened to these patients. It would be helpful for the authors to present some outcomes data including percent successfully weaned, percent with ongoing ventilation, percent died, percent transferred back to a short term acute care hospital, percent discharged home etc. It would also be helpful to understand patients' functional status if the data is available. Such descriptions would be helpful to truly quantify not only the burden of disease but the likelihood of recovery.

2. The authors' data indicates that $51.2 \%$ of post COVID-19 patients required MV and $22 \%$ required RRT of some form. It is well known that during the first spike in COVID in the spring, there was a substantial drop in other disease states presenting to the hospital. In essence, for a while most hospitals were seeing COVID and nothing but COVID. I am unclear as to how much this was true in Southern California but it is a phenomenon seen in multiple parts of the country. Therefore, comparing the COVID patients to the non-COVID patients during the same time may not be the best comparison. It would be interesting, if possible, to compare the COVID-19 patients admitted during the study period to patients admitted the year prior in terms of rates of MV, HD, tracheostomy, LOS at originating hospital, etc. This would provide broader context for what is truly COVID related.

\section{Minor Comments}

1. In the Abstract it is stated "Median age: 68 [44-94] years, $61 \%$ male, $80.5 \%$ with tracheostomy, $51.2 \%$ on invasive mechanical ventilation, $22 \%$ receiving hemodialysis". It is confusing as to whether $80.5 \%$ of the 41 patients had a tracheostomy or $80.5 \%$ of patients who were receiving MV had a tracheostomy. Please clarify.

2. In the Methods it states "Missing data were omitted from analysis. For the variables, serum albumin and serum glucose, $n=36$ and $n=40$ respectively." I am not sure what is meant by these sentences. Please clarify.

Is the work clearly and accurately presented and does it cite the current literature? Yes

Is the study design appropriate and is the work technically sound? Yes

Are sufficient details of methods and analysis provided to allow replication by others? Yes

If applicable, is the statistical analysis and its interpretation appropriate? Yes

Are all the source data underlying the results available to ensure full reproducibility? No

Are the conclusions drawn adequately supported by the results? Yes

Competing Interests: No competing interests were disclosed. 
Reviewer Expertise: Critical care outcomes, shared decision-making, prolonged mechanical ventilation

I confirm that I have read this submission and believe that I have an appropriate level of expertise to confirm that it is of an acceptable scientific standard, however I have significant reservations, as outlined above.

Author Response 12 Apr 2021

Meg Hassenpflug, Barlow Respiratory Hospital, Los Angeles, USA

We kindly thank the Reviewer for his thoughtful comments and suggestions for our paper. Our responses follow:

Major Comments

We absolutely agree that what happens to these patients (outcomes) is of foremost interest. Referring to the study inclusion dates, this group of patients represents the beginning of the COVID pandemic in southern California. Our central purpose at this relatively early stage of the pandemic was to quickly share with critical care providers the characterization of the population of post-COVID infection patients admitted to our facility, to underscore the important role of the LTAC in responding to the COVID19 crisis. It was not our intent to present outcomes at this early timepoint. With a small cohort of patients, and only 14 patients admitted for ventilator weaning, we believe that attempting to present any outcomes data may have been misleading. We agree that it would be helpful to understand patients' functional status relative to a variety of specific outcomes (our interpretation of the phrase "likelihood of recovery"). These data were not included in the initial characterization of the population as it was not our intent to present any outcomes analysis at this early timepoint.

The Reviewer's point on the COVID vs non-COVID patient populations is well taken. The only minor comparison mentioned in the text between the two groups was length of stay (LOS) at the transferring hospital. An oversight on our part in the Abstract and Results is that we failed to include those data. We regret the omission and have excluded the related statement from the Abstract, and added the data to Results in the revised text. The LOS at the transferring acute care hospital was median 42 [8-78] days for the post-COVID pneumonia cohort, and median 16 [1-96] days for non-COVID patients admitted during the same time period. We made no attempt to interpret the data, and considered this simply an interesting informational contrast to be made at that point in time. We very much appreciate your suggestion to compare the COVID-19 patients admitted during the study period to patients admitted the year prior in terms of rates of MV, HD, tracheostomy, LOS at originating hospital, etc, and are doing so as we build on this effort. Thank you.

Minor Comments

1. In the Abstract, the Reviewer asks for clarification regarding whether $80.5 \%$ of the 41 patients had a tracheostomy or $80.5 \%$ of patients who were receiving MV had a tracheostomy. The percentages refer to the entire cohort, but we see where confusion could arise. The Abstract has been edited for clarity. 
2. The Reviewer asks for clarification of statements regarding missing data and the various " $n$ " for the laboratory values serum albumin and serum glucose. We were informing the reader that these two variables presented in Table 2 were not reflective of the entire cohort of 41 patients due to a few missing values. The text has been revised.

Competing Interests: No competing interests were disclosed.

The benefits of publishing with F1000Research:

- Your article is published within days, with no editorial bias

- You can publish traditional articles, null/negative results, case reports, data notes and more

- The peer review process is transparent and collaborative

- Your article is indexed in PubMed after passing peer review

- Dedicated customer support at every stage

For pre-submission enquiries, contact research@f1000.com 